\title{
COMPUTATIONAL MODELING OF A REGULAR WAVE TANK
}

\author{
M. N. Gomes ${ }^{a}$, \\ C. R. Olinto ${ }^{\mathrm{a}}$, \\ L. A. O. Rocha ${ }^{a}$, \\ J. A. Souza ${ }^{a}$, \\ and $L$. A. Isoldi ${ }^{\mathrm{a}}$ \\ ${ }^{a}$ Universidade Federal do Rio Grande \\ Programa de Pós-Graduação em Modelagem \\ Computacional \\ Av. Itália, km 8, Bairro Carreiros \\ CEP 96.201-900, Rio Grande, Brasil \\ liercioisoldi@furg.br
}

\section{ABSTRACT}

This paper presents two different numerical methodologies to generate regular gravity waves in a wave tank. We performed numerical simulations of wave generation through the FLUENT ${ }^{\mathbb{B}}$ package, using the Volume of Fluid (VOF) multiphase model to reproduce the wave propagation in the tank. Thus it was possible to analyze two methods for generating regular waves that could be used in future work, especially in the study of devices of energy conversion from ocean waves into electrical energy.

Keywords: Regular waves, wave tank, Volume of Fluid (VOF), numerical simulation, FLUENT ${ }^{\circledR}$.

\section{NOMENCLATURE}

A

$\mathrm{F}$

$\mathrm{g}$

$\mathrm{h}$

\section{$\mathrm{H}$}

$\mathrm{k}$

$\mathrm{L}$ amplitude, $\mathrm{m}$ external forces, $\mathrm{N}$ acceleration due to gravity, $\mathrm{m} / \mathrm{s}^{2}$ depth , m

height, $m$ wave number, $\mathrm{m}^{-1}$ wavelength, $\mathrm{m}$ coordinate normal to the bottom mass transfer phase $\mathrm{q}$ to phase $\mathrm{p}, \mathrm{kg} / \mathrm{s}$ mass transfer phase $\mathrm{p}$ to phase $\mathrm{q}, \mathrm{kg} / \mathrm{s}$ unit exterior normal vector static pressure, $\mathrm{Pa}$ piston displacement, $\mathrm{m}$ maximum displacement the piston, $\mathrm{m}$ source term

time, $s$ wave period, s velocity component in $\mathrm{x}, \mathrm{m} / \mathrm{s}$ vector velocity, $\mathrm{m} / \mathrm{s}$ horizontal position, $\mathrm{m}$ vertical direction velocity component in $\mathrm{y}, \mathrm{m} / \mathrm{s}$

\section{Greek symbols}

$\eta \quad$ elevation of the wave, $\mathrm{m}$

$\rho$ density, $\mathrm{Kg} / \mathrm{m}^{3}$

$\phi \quad$ potential function

$\mu \quad$ viscosity, $\mathrm{kg} / \mathrm{m} . \mathrm{s}$

$\omega \quad$ circular frequency of wave

\section{INTRODUCTION}

Countries have recently invested in the exploration of new energy sources, especially in the so-called renewable sources. And one of those that has been highlighted is the energy contained in ocean waves. According to Cruz and Sarmento (2004), the oceans, containing the biggest of all natural resources, have a huge energy potential, which can contribute significantly to the growing energy needs at a global level.

One way to investigate this potential is through computer simulations and experiments in laboratories. For this we use models that physically describe the phenomenon. To analyze devices capable of converting the energy of sea waves into electrical energy it is important to master the different theories of gravity waves and the generation process thereof.

In this work two different ways of generating regular gravity waves, in a numerical wave tank, were developed through computer simulations. We used a two-dimensional numerical model that is based on the Volume of Fluid (VOF) multiphase model , using techniques similar to Liu et al (2007), Repalle, et al (2007), and Horko (2007). Both the process of numerical simulation and the analysis of the results used the package of computational fluid dynamics (CFD) software composed of FLUENT ${ }^{\circledR}$ and GAMBIT ${ }^{\circledR}$. To familiarize the reader with the subject, a short study on two types of wave theories (linear and nonlinear) was initially presented.

\section{WAVE THEORY}

Here, gravity waves (also called surface waves) were studied, in special the regular waves. Gravity 
waves are understood to be regular waves of welldefined behavior and constant in each time period.

Two theories of gravity waves were discussed: linear wave theory that applies to waves with small amplitude and length and Stokes 2nd Order Nonlinear theory which applies to waves with large amplitudes and lengths.

According to McCormick (1976) waves at the free surface exist because of the natural tendency of fluids is to remain in equilibrium. When an object is dropped into a tank of water a disturbance is created and forms a surface wave. Subsequent moves on the surface are a result of gravity, tending to return the water to its position without disturbance. Since these waves are the result of gravity they are called gravity waves. According to Dean and Dalrymple (1991), another factor that can cause a disturbance in free water surface is the action of the winds. Furthermore, waves can occur in all sizes and shapes, depending on the magnitude of the forces acting on the water. In Fig. 1, it is possible to observe the main characteristics of the waves.

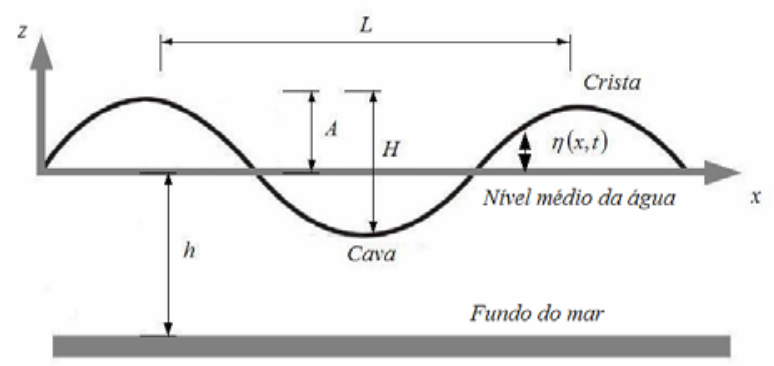

Figure 1. Characteristics of wave.

According to Fig. 1, $\mathrm{h}$ is the depth (distance from the seabed to the mean sea level), $\mathrm{A}$ is the amplitude (distance between crest and the mean sea level) $\mathrm{H}$ is the height (distance between cava and crista ) $\eta$ is called the elevation of the wave and $L$ is the wavelength (distance between two successive crests or two troughs) that can be calculated by the following equation:

$$
L=\frac{g T^{2}}{2 \pi} \tanh \left(\frac{2 \pi h}{L}\right)
$$

where $\mathrm{g}$ is the acceleration due to gravity and $\mathrm{T}$ is the wave period.

The period of a wave is defined as the time required, for the wave form, describes a complete cycle, in this case a wavelength.

All waves, gravity, acoustic or electromagnetic, obey a form of wave equation. The dependent variable in each case depends on the physical phenomenon and boundary conditions which may be linear or nonlinear. The flow of an incompressible surface wave must satisfy the special linear wave equation known as Laplace's equation, if the flow is assumed irrotational (McCormick, 1976).
Gravity waves in the water must obey the following three boundary conditions:

Free surface kinematic condition: water particles can not cross the free surface. To satisfy the condition of particle velocity $Z=\eta$ must be equal to the normal speed at the free surface. Mathematically condition is:

$$
\left.V\right|_{Z=\eta} \approx \frac{\partial \eta}{\partial t} k=\left.\frac{\partial \phi}{\partial Z}\right|_{Z=0} k
$$

where $\phi$ is a potential function and $\mathrm{k}$ is the normal unit vector.

Free surface dynamic condition: the pressure at the free surface is zero for any position $x$ and time $t$. Basically consists of applying the Bernoulli equation to the free surface and gets:

$$
\frac{\partial \phi}{\partial t}+g \eta+\frac{1}{2} V^{2}=0
$$

where $\phi$ is a potential function, $g$ is gravity, $\eta$ is the elevation of free surface and $\mathrm{V}$ the velocity. The second boundary condition can be linearized using the condition of dynamic linearization given by:

$$
\eta=-\left.\frac{1}{g} \frac{\partial \phi}{\partial t}\right|_{Z=\eta}
$$

Bottom condition: the bottom can be considered as horizontal and impermeable. Mathematically, in $\mathrm{z}=-\mathrm{h}$ :

$$
V . m=\frac{\partial \phi}{\partial N}=0
$$

where $\mathrm{m}$ is the coordinate normal to the bottom and $\mathrm{N}$ is the unit exterior normal vector.

We are dealing with a continuum fluid inside the wave, so the continuity equation is satisfied. If the flow is irrotational, the velocity can be represented by a potential function, so the continuity equation can be written as follows:

$$
\nabla^{2} \phi=0
$$

where a solution to Eq. (6) satisfying the boundary conditions presented provides the following velocity potential:

$$
\phi=\frac{a g}{\omega} \frac{\cosh (k z+k h)}{\cosh (k h)} \operatorname{sen}(k x-\omega t)
$$


From Eq. (7) the following components for speed are obtained:

$$
\begin{gathered}
u=\frac{\partial \phi}{\partial x}=\frac{a g k}{\omega} \frac{\cosh (k z+k h)}{\cosh (k h)} \cos (k x-\omega t) \\
w=\frac{\partial \phi}{\partial z}=\frac{a g k}{\omega} \frac{\operatorname{senh}(k z+k h)}{\cosh (k h)} \operatorname{sen}(k x-\omega t)
\end{gathered}
$$

where $\mathrm{u}$ is the velocity component in $\mathrm{x}$ direction and $\mathrm{w}$ is the velocity component in $\mathrm{z}$ direction.

An equation describing the motion of the free surface can also be highlighted:

$$
\eta=A \cos (k x-\omega t)
$$

The other theory mentioned above, the theory of Stokes, consists in assuming that the properties of wave motion, as the velocity potential, can be represented by a series of small perturbations (McCormick, 1976). The same applies for greater wave heights in deeper waters. In this theory the velocity potential is obtained by a power series of type:

$$
\phi=\varepsilon \phi_{1}+\varepsilon^{2} \phi_{2}+\varepsilon^{3} \phi_{3}+\ldots \ldots
$$

The solution containing the first-order term $\phi_{1}$ represents the linear theory. The expansion to the second term $\phi_{2}$ represents Stokes second order theory and so on.

Unlike what occurs in the linear theory, the trajectory of a particle when we consider non-linear theories, does not describe a closed orbit. This behavior approaches even more the behavior of real waves with the movement of ocean currents, indicating the mass transfer along the direction of wave propagation.

Thus the equation describing the motion of the free surface in Stokes second order theory is given by:

$$
\begin{aligned}
& \eta=A \cos (k x-\omega t)+\ldots \ldots \\
& \ldots+\frac{A^{2} k \cosh (k h)}{4 \operatorname{senh}^{3}(k h)}[2+\cosh (2 k h)] \cos 2(k x-\omega t)
\end{aligned}
$$

And also the velocity components are:

$$
\begin{aligned}
u=A g k \frac{\cosh (k z+k h)}{\omega \cosh (k h)} \cos (k x-\omega t)+ \\
A^{2} \omega k \frac{\cosh 2 k(k+z)}{\operatorname{sen}^{4}(k h)} \cos 2(k x-\omega t)
\end{aligned}
$$

$$
\begin{aligned}
& w=\operatorname{Agk} \frac{\operatorname{senh}(k z+k h)}{\omega \operatorname{senh}(k h)} \operatorname{sen}(k x-\omega t)+ \\
& A^{2} \omega k \frac{\operatorname{senh} 2 k(k+z)}{\cos ^{4}(k h)} \operatorname{sen} 2(k x-\omega t)
\end{aligned}
$$

where $\mathrm{z}$ is the position variation of the free water surface to the seabed. Further details of this theory can be found in McCormick (1976) and Dean and Dalrymple (1991).

\section{WAVE TANK}

One purpose of this paper is to discuss numerical simulations using two different ways of generating surface waves on a tank (mobile and function methodologies). Some computer simulations of these processes, for various boundary conditions, have been developed. In Fig 2 the wave tank is shown schematically.

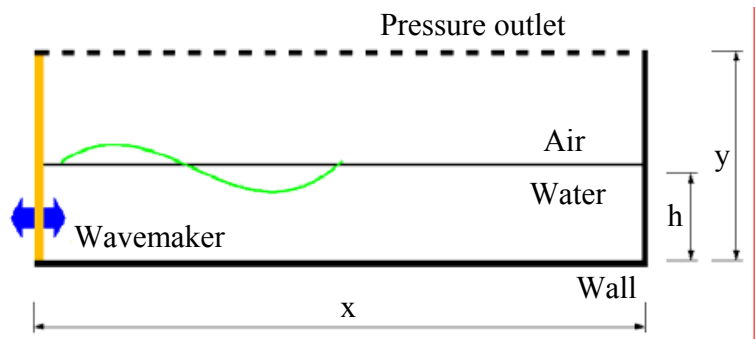

Figure 2. Schematic representation of the wave tank.

It is possible to observe in Figure 2, that on top of the wave tank (pressure outlet) the boundary condition of atmospheric pressure is applied; and at the boundary condition (called wall) the velocity is considered zero. As for the wave generator, the boundary condition depends on of one of two methods chosen.

Mobile Methodology: This methodology consists in applying to the wave generator the boundary condition of mobile wall, which will move similarly to a piston. For this is used the dynamic mesh technique that allows to employ a variation of velocity to the mobile wall via a User Defined Function (UDF).

The movement control of the piston (mobile wall) is made according to the characteristics of the wave that will be generated, being necessary to know the period and the wave height of this wave. Then, using the transfer function, which relates the wave height and piston displacement, it is possible to determine the displacement that the piston needs to do to generate the wave with the desired characteristics. This transfer function is defined by (Dean and Dalrymple, 1991):

$$
\frac{H}{S}=\frac{2(\cosh (2 k h)-1)}{\sinh (2 k h)+2 k h}
$$


where $\mathrm{H}$ is the height of wave to be generated, $\mathrm{S}$ is the piston displacement, $\mathrm{h}$ is depth of wave tank and $\mathrm{k}$ is the wave number that is given by $k=2 \pi / L$, where $\mathrm{L}$ is the wavelength.

Knowing this information it is possible to determine the equation that controls the movement of the mobile wall. This motion equation is defined by (Liu et al, 2008):

$$
x(t)=\frac{S_{0}}{2}\left(1-e^{-\frac{5 t}{2 T}}\right) \sin (\omega t)
$$

where $S_{0}$ is the maximum displacement of the piston (wave generator), $\mathrm{T}$ is the period of the wave and $\omega$ its frequency, given by $\omega=2 \pi / t$. But since the goal is to apply the velocity condition to the piston, then derive Eq. (16) as a function of time and get the piston velocity (wave generator), given by:

$$
v(t)=\left(\frac{S_{0}}{2}\right)\left[\left(1-e^{-\frac{5 t}{2 T}}\right) \omega \cos (\omega t)+\frac{5}{2 T} e^{-\frac{5 t}{2 T}} \sin (\omega t)\right](17)
$$

Function Methodology: This methodology, developed in Horko (2007), consists of applying to the wave generator an entry velocity (velocity inlet) through a User Defined Function (UDF). The velocity varies according to Eq. (13) and Eq. (14), i.e., applies to a wave generator a velocity variation according to Stokes second order theory.

\section{NUMERICAL MODEL}

The numerical model used is based on the Volume of Fluid (VOF) method, which allows to computationally simulate a wave tank by observing the interaction between water and air in a manner very close to reality, as in FLUENT (2005).

The VOF method can model two or more immiscible fluids by solving a single set of equations for the amount of movement and tracking the volume fraction of each fluid throughout the volume. The VOF formulation relies on the fact that two or more phases are impenetrable. For each additional step added to the model, the volume fraction is calculated on the volume. In each control volume, the sum of the volume fraction of all phases is unitary. The fields for all variables and properties are shared by the phases and represent volume averaged values, as long as the volume fraction of each phase is known locally. Thus, the variables and properties in a given volume represent one of the phases or a mixture of phases, depending on the values of volume fraction. In other words, if the q-th fluid volume fraction is denoted as $\alpha_{\mathrm{q}}$, then the following three conditions are possible:

$$
\begin{aligned}
& \alpha_{q}=0 \text { : The volume is empty (of q-th fluid); } \\
& \alpha_{q}=1: \text { The volume is full (of q-th fluid); } \\
& 0<\alpha_{q}<1: \text { The volume contains the interface }
\end{aligned}
$$

between the q-th fluid and one or more fluids;

The numerical model employed consists basically in the continuity equation for the fraction of one or more phases :

$$
\frac{1}{\rho q}\left[\frac{\partial}{\partial t}\left(\alpha_{q} \rho_{q}\right)+\nabla\left(\alpha_{q} \rho_{q} \vec{v}_{q}\right)=S_{\alpha_{q}}+\sum_{p=1}^{n}\left(\dot{m}_{p q}-\dot{m}_{q p}\right)\right](18)
$$

where $\dot{m}_{q p}$ is the mass transfer phase $\mathrm{q}$ to phase $\mathrm{p}$, $\dot{m}_{p q}$ is the mass transfer phase $\mathrm{p}$ to phase $\mathrm{q}, S_{\alpha_{q}}$ is the source term (in this case zero) and $\rho$ is the density.

And also for the momentum equation:

$$
\begin{aligned}
\frac{\partial}{\partial t}(\rho \vec{v})+\nabla(\rho \vec{v} \vec{v}) & = \\
& -\nabla p+\nabla \cdot\left[\mu\left(\nabla \vec{v}+\nabla \vec{v}^{T}\right)\right]+\rho \vec{g}+\vec{F}
\end{aligned}
$$

where $\mathrm{p}$ is the static pressure, $\rho \vec{g}$ is the gravitational force, $\vec{F}$ are external forces, $\mu$ is the viscosity.

The validation of the implementation of VOF model for this type of problem is presented below, along with a case study where the waves generated are compared numerically with those obtained by analytical equations of motion of the free surface.

\section{NUMERICAL SIMULATIONS}

The proposed case study is to analyze numerically and graphically the process of generation of waves with different characteristics. Four simulations for each method of wave generation were performed, as shown in Table 1. For convention, MM for mobile methodology and MF for function methodology were adopted, $\mathrm{H}$ the wave height, $\mathrm{T}$ the period, $h$ depth of the tank, $\mathrm{L}$ wave length and $\mathrm{P}$ the size of the regular square mesh used.

Table 1. Simulations performed.

\begin{tabular}{ccccccc}
\hline & Method & $\mathrm{H}(\mathrm{m})$ & $\mathrm{T}(\mathrm{s})$ & $\mathrm{h}(\mathrm{m})$ & $\mathrm{L}(\mathrm{m})$ & $\mathrm{P}(\mathrm{m})$ \\
\hline 1 & MF & 1.000 & 6.000 & 16.000 & 53.60 & 0.100 \\
2 & MF & 0.200 & 1.130 & 1.200 & 2.000 & 0.010 \\
3 & MF & 0.140 & 0.800 & 0.500 & 1.020 & 0.005 \\
4 & MF & 0.140 & 0.800 & 0.500 & 1.020 & 0.010 \\
5 & MM & 0.140 & 0.800 & 0.500 & 1.020 & 0.010 \\
6 & MM & 1.000 & 6.000 & 16.000 & 53.60 & 0.100 \\
7 & MM & 0.140 & 1.500 & 1.000 & 3.400 & 0.010 \\
8 & MM & 0.140 & 1.500 & 0.500 & 2.800 & 0.010 \\
\hline
\end{tabular}


The simulations were all performed in the laminar regime, adopting PISO algorithm for the pressure-velocity coupling. In all cases was used a regular grid formed by square-type finite volume, it is important to note that in the MM method one of the impositions is to use a mesh with these features.

In simulation 1 was carried out the comparison of the numerical wave with those generated by Eq. (12). For this we used a tank with $200 \mathrm{~m}$ long and $16 \mathrm{~m}$ depth, similar to that used in Liu et al. (2008). In Fig. 3 it is possible to observe the good agreement of results in a vertical line at position $\mathrm{x}=20 \mathrm{~m}$, inside the wave tank, where the absolute maximum difference after the stabilization of the wave was $1.2 \%$. It can still be seen that the portion referring to the term of the expansion of Stokes second order theory is irrelevant to medium waves with characteristics such as those of case one.

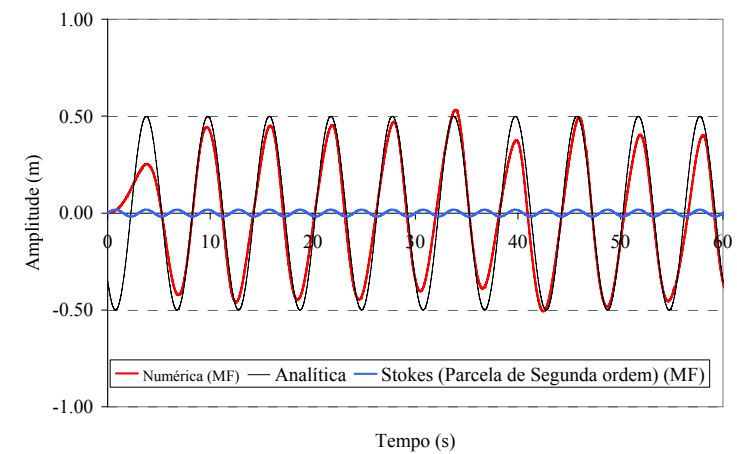

Figure 3. Amplitude of the wave of case 1 at position

$$
\mathrm{x}=20 \mathrm{~m} \text {. }
$$

In simulation 2 a tank with a length of $15 \mathrm{~m}$ and 1.2 m depth similar to Repalle et al. (2007) was used. In this case the velocity profile in the $\mathrm{x}$ direction was analyzed through vertical lines drawn inside the tank. Figure 4 presents a comparison of the moment at $\mathrm{t}=16 \mathrm{~s}$ at position $\mathrm{x}=4 \mathrm{~m}$ between the numerical solution and Eq. (13), showing that the characteristics of velocity is maintained throughout the wave tank. The analysis is performed only in the region that is filled with water, where the average level of water is $1.2 \mathrm{~m}$ and is considered as a reference, i.e. zero. Elsewhere it is noted that the farther away the measuring line of the wave generator the analytical and numerical results have better agreement due to the velocity profile in this region being already stabilized.

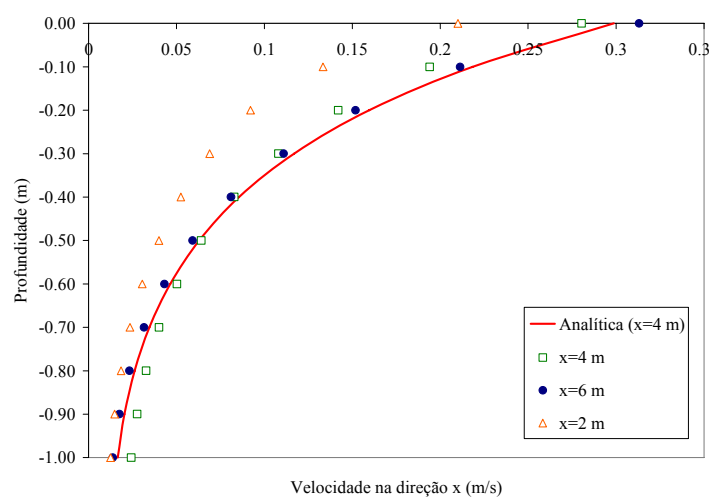

Figure 4. Velocity profile toward the simulation 2.

In cases 3 and 4, numerical simulations of wave generation were carried out with the same characteristic in a wave tank $15 \mathrm{~m}$ long and $0.5 \mathrm{~m}$ deep, in which only the number of volumes of the mesh was modified. In case 3 it has 480,000 volumes and in case 4 has 120,000 volumes. It was observed that the mesh of case 4 is already a good standard, because when they refine the same in case 3 the absolute maximum margin of error in generating the wave remains approximately $12 \%$. Figure 5 presents the interaction between water (red) and air (blue) in the wave tank in case 4 , at time $t=16 \mathrm{~s}$, showing the ability of the proposed numerical model to generate regular waves.

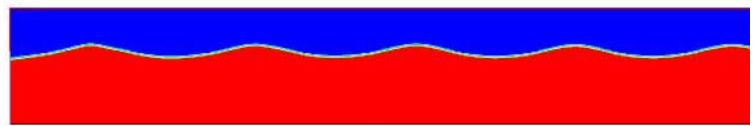

Figure 5. Generation wave with the VOF model in case 4 .

In Fig. 6 the topology of the velocities in the $x$ direction is shown, in case 4 , at time $t=16 \mathrm{~s}$, and in Fig. 7 presents a similar ilustration, but for velocities in the y direction.

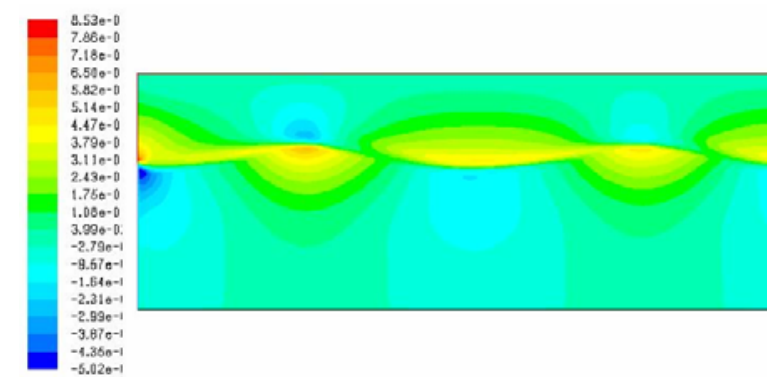

Figure 6. Topology of velocity in x direction. 


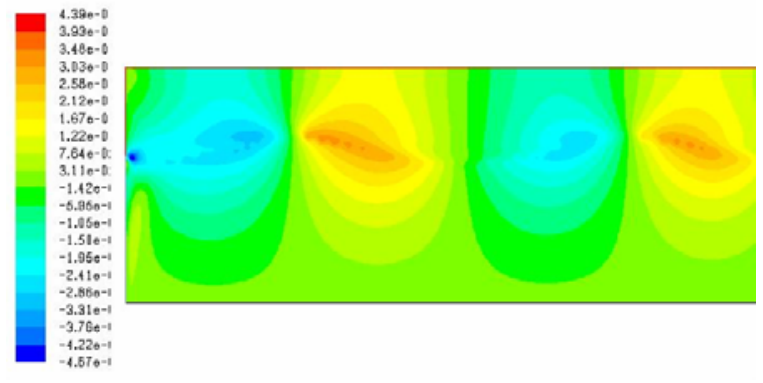

Figure 7. Topology of velocity in the y direction.

From case 5, simulations with the methodology of the mobile mesh were performed, analyzing the velocity at different positions along the wavelength. For according to CEM (2002) velocities over a wavelength should have characteristics similar to those shown in Fig. 8.

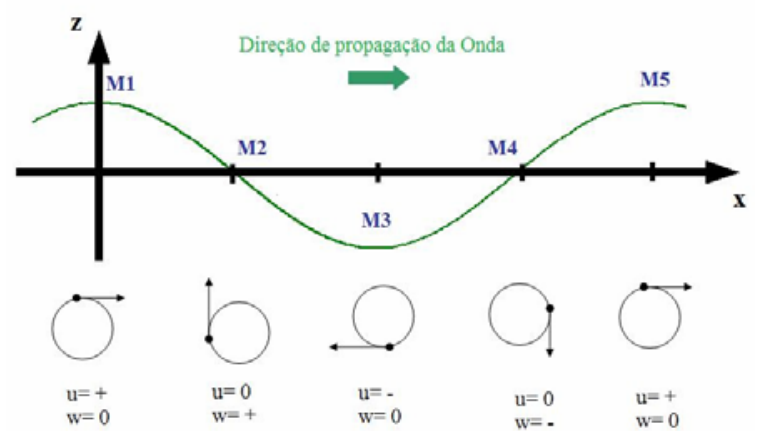

Figure 8. Characteristic of the wave velocity along a wavelength.

Still considering Fig. 8, it is observed a good approximation of the characteristics of velocities obtained by numerical simulation with the characteristics proposed by the theory, as shown in Fig. 9 and Fig. 10.

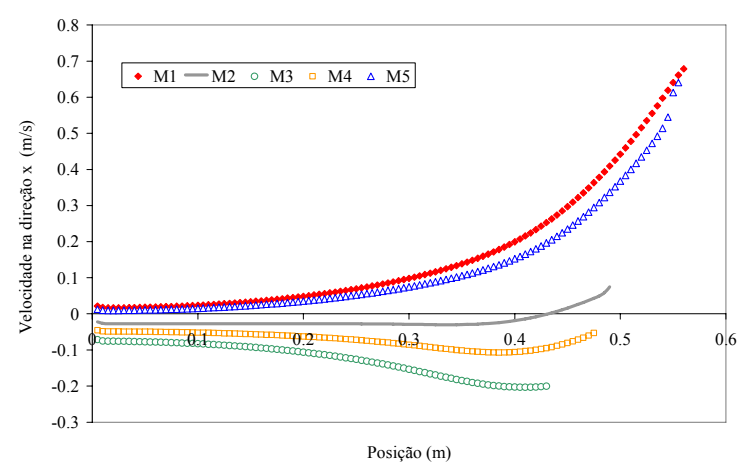

Figure 9. Velocity in $\mathrm{x}$ direction along a wave.

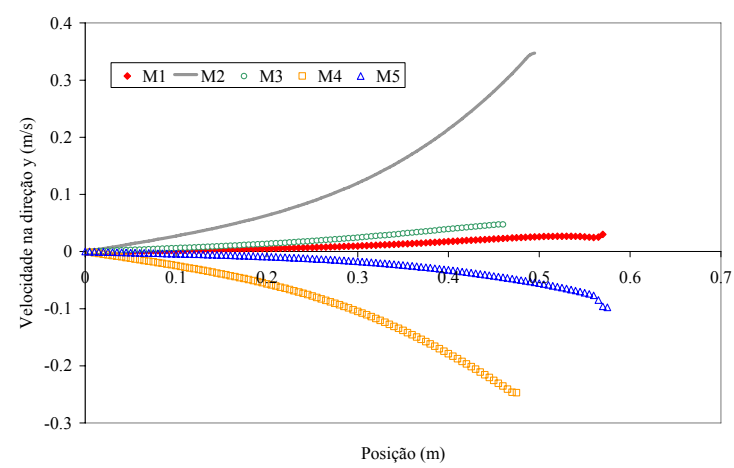

Figure 10 . Velocity in the y direction along a wave.

Please note that as the measurement process takes into account the elevation of free water surface, as in Fig 8, the curves presented in Fig. 9 and Fig. 10 have different sizes because each measuring line is filled with water at different levels.

In the simulation 6 the ability of the numerical model, with the methodology of mobile mesh, to generate waves of medium size is tested. Figure 11 represents the wave amplitude at position $\mathrm{x}=20 \mathrm{~m}$ in a wave tank with the same characteristics of case 1 . It is possible to observe that the model is effective with a maximum absolute difference of $0.5 \%$.

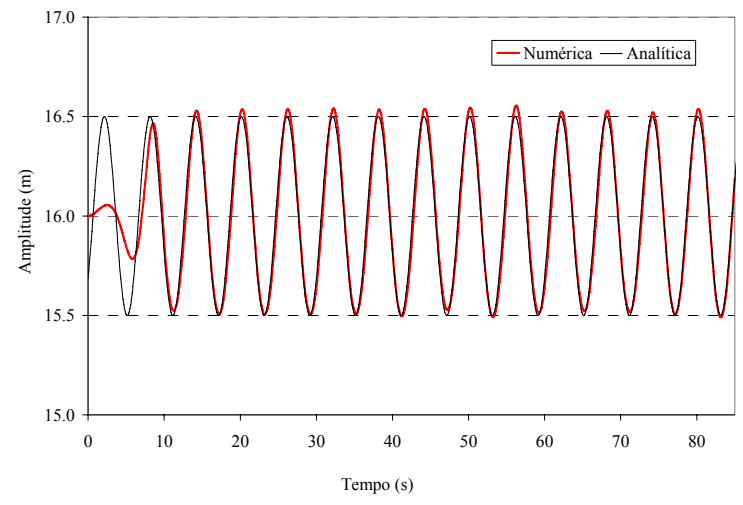

Figure 11. Magnitude of the wave at $\mathrm{x}=20 \mathrm{~m}$ for case 6 .

Finally, in cases 7 and 8, numerical simulations of the generation of waves with the same period on a wave tank with a length of $15 \mathrm{~m}$ and $1 \mathrm{~m}$ depth for case 7 and $0.5 \mathrm{~m}$ for case 8 were carried out. As it can be noted in Fig. 12, in simulation 7 the bottom has an influence on the generation of the wave, thus observing Eq. (1), the wavelength has just been changing. It can be observed in Fig 12, a good agreement of results with the analytic solution, but in case 8 it is clear the effect of bottom on the generation of the wave since the depth is about five times smaller than the wavelength and the recommended, according to McCormick (1976), is a half. In case 7 the wave is influenced by the bottom of the tank, but obeying a certain regularity on the axis $\mathrm{x}$. The absolute maximum difference found in these two simulations was around $5 \%$. 


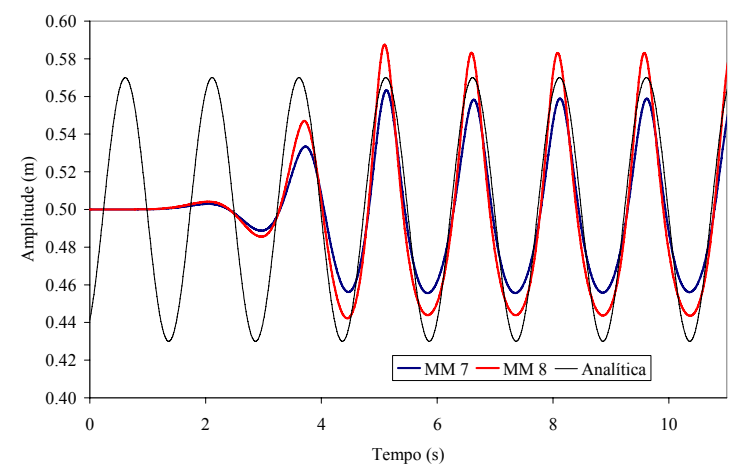

Figure 12. Magnitude of the wave in cases 7 and 8.

\section{CONCLUSIONS}

Analyzing the results obtained in the eight simulations developed in this work means that the computational modeling approach, based on the VOF model is appropriate, given the fact that we can see that it generates waves with the desired characteristics, with maximum differences of about $5 \%$, comparing the numerical solution with the analytical.

The two methods of generating wave present similar results, with the only parameter that distinguishes them in a relevant way is the computational processing time, which is more advantageous to employ the function methodology that uses about $75 \%$ of the time used by the mobile methodology.

Therefore it was possible to validate these numerical simulation methods for the generation of regular waves, comparing their results with the analytic solution of this problem, showing that the VOF model is a tool able to reproduce adequately the interaction between water and air.

Thus, this study contributes to future work in the area of computational modeling of energy transformation of waves into electrical energy. For example, in numerical simulation of Oscillating Water Column (OWC) and Overtopping devices, where it is needed to generate regular waves of gravity in order to analyze the behavior of the power converter due to the incidence of the wave in it.

\section{ACKNOWLEDGEMENTS}

The authors thank CAPES for the financial support provided to this work.

\section{REFERENCES}

CEM, 2002, Coastal Engineering Manual, Army Corps of Engineers, U.S., Washington.

Cruz, J. M. B. P., Sarmento, A. J. N. A., 2004, Energia das Ondas: Introdução aos Aspectos Tecnológicos, Económicos e Ambientais, Ed. Instituto do Ambiente, Portugal, Amadora. (in Portuguese)
Dean, R. G., and Dalrymple, R. A., 1991, Water wave mechanics for engineers and scientists, vol. 2, World scientific, Singapore.

Fluent Inc., 2005, FLUENT 6.2 user's guide.

Horko, M., 2007, CFD Optimisation of an Oscillating Water Column Energy converter, Thesis of Master of Engineering Science, School of Mechanical Engineering, The University of Western, Australia.

Liu, E., 2008, Aplication of Numerical Wave Tank to OWC air chamber for wave energy conversion, Procedings of the Eighteenth International Offshore and Polar Engineering Conference, Vancouver, Canada.

McCormick, M. E., 1976. Ocean engineering wave mechanics, John Wiley \& Sons, USA, New York.

Repalle, N, 2007, CFD simulation of wave runup on a spar cylinder, Proceedings 16th Australasian Fluid Mechanics Conference, Gold Coast, Australia.

Received: August 07, 2008

Revised: September 07, 2008 Accepted: October 07, 2008 